\title{
Presence and extent of cardiac computed tomography angiography defined coronary artery disease in patients presenting with syncope
}

\author{
S. Altintas ${ }^{1}$ T. Dinh ${ }^{1}$ N. G. H. M. Marcks ${ }^{1,2}$ - M. Kok ${ }^{2,3}$ - A. J. J. Aerts ${ }^{4}$ B. Weijs ${ }^{1}$ Y. Blaauw ${ }^{5}$ \\ J. E. Wildberger ${ }^{2,3}$ • M. Das ${ }^{2,3}$ • B. L. J. H. Kietselaer ${ }^{1,2,3}$ • H. J. G. M. Crijns ${ }^{1,2}$
}

Published online: 20 March 2017

(c) The Author(s) 2017. This article is available at SpringerLink with Open Access.

\begin{abstract}
Background In syncope patients, presence of coronary artery disease $(\mathrm{CAD})$ is associated with poor prognosis. However, data concerning CAD prevalence in syncope patients without known cardiovascular disease are lacking. Therefore, the aim of this study was to investigate presence and extent of CAD in syncope patients.

Methods We included 142 consecutive patients presenting with syncope at the outpatient cardiology clinic who underwent coronary computed tomography (CT) angiography. Syncope type was ascertained by two reviewers, blinded for coronary CT angiography results. Of the patients, $49 \mathrm{had}$ cardiac syncope (arrhythmia or structural cardiopulmonary disease) and 93 had non-cardiac syncope (reflex [neurallymediated], orthostatic or of unknown cause). Cardiac syncope patients were compared with matched stable chest
\end{abstract}

S. Altintas and T. Dinh contributed equally.

Electronic supplementary material The online version of this article (doi: 10.1007/s12471-017-0970-7) contains supplementary material, which is available to authorized users.

H. J. G. M. Crijns

hjgm.crijns@mumc.nl

1 Department of Cardiology, Maastricht University Medical Center+ (MUMC+), Maastricht, The Netherlands

2 Cardiovascular Research Institute Maastricht (CARIM), School for Cardiovascular Diseases, MUMC+, Maastricht, The Netherlands

3 Department of Radiology, MUMC+, Maastricht, The Netherlands

4 Department of Cardiology, Zuyderland Medical Center, Heerlen, The Netherlands

5 Department of Cardiology, University Medical Center Groningen, Groningen, The Netherlands pain patients regarding age, gender, smoking status, diabetes mellitus type 2 and systolic blood pressure.

Results Distribution of CAD presence and extent in cardiac and non-cardiac syncope patients was as follows: $72 \%$ versus $48 \%$ any $\mathrm{CAD} ; 31 \%$ versus $26 \%$ mild, $8 \%$ versus $14 \%$ moderate and $33 \%$ versus $7 \%$ severe $\mathrm{CAD}$.

Compared with non-cardiac syncope, patients with cardiac syncope had a significantly higher CAD presence and extent $(p=0.001)$. Coronary calcium score, segment involvement and stenosis score were also higher in cardiac syncope patients ( $p$-values $\leq 0.004)$. Compared to the chest pain control group, patients with cardiac syncope showed a higher, however, non-significant, prevalence of any CAD (72\% versus $63 \%$ ) and severe CAD (33\% versus $19 \%$ ).

Conclusion Patients with cardiac syncope show a high presence and extent of CAD in contrast to non-cardiac syncope patients. These results suggest that CAD may play an important role in the occurrence of cardiac syncope.

Keywords Coronary artery disease $\cdot$ Multidetector computed tomography $\cdot$ Cardiogenic syncope

\section{Introduction}

Syncope is defined as a transient loss of consciousness due to transient global cerebral hypoperfusion and is characterised by a rapid onset, short duration and complete spontaneous recovery $[1,2]$. It concerns a common medical problem, with an incidence rate of 6.2 per 1000 person-years for a first report [3]. Syncope is a symptom with a wide spectrum of potential aetiologies for which accurate diagnosis, using additional testing, is of high importance [4-6]. Along with the search for the underlying diagnosis, defining prog- 
Table 1 Baseline characteristics of the study population

\begin{tabular}{|c|c|c|c|c|c|c|}
\hline \multirow[b]{2}{*}{ Patient characteristics } & \multicolumn{3}{|c|}{$\begin{array}{l}\text { Cardiac syncope vs non-cardiac syncope } \\
n=142\end{array}$} & \multicolumn{3}{|c|}{$\begin{array}{l}\text { Cardiac syncope vs chest pain control group } \\
n=147\end{array}$} \\
\hline & $\begin{array}{l}\text { Cardiac syn- } \\
\text { cope } \\
n=49\end{array}$ & $\begin{array}{l}\text { Non-cardiac } \\
\text { syncope } \\
n=93\end{array}$ & $P$-value & $\begin{array}{l}\text { Cardiac syn- } \\
\text { cope } \\
n=49\end{array}$ & $\begin{array}{l}\text { Chest pain } \\
\text { control group } \\
n=98\end{array}$ & $P$-value \\
\hline Age, years & $60 \pm 13$ & $54 \pm 12$ & 0.002 & $60 \pm 13$ & $60 \pm 12$ & 0.810 \\
\hline Male gender & $32(65)$ & $57(61)$ & 0.716 & $32(65)$ & $64(65)$ & $>0.999$ \\
\hline BMI, $k g / m^{2}$ & $26 \pm 3$ & $26 \pm 4$ & 0.365 & $26 \pm 3$ & $27 \pm 4$ & 0.116 \\
\hline Systolic Blood Pressure, $\mathrm{mm} \mathrm{Hg}$ & $143 \pm 20$ & $140 \pm 21$ & 0.349 & $143 \pm 20$ & $144 \pm 16$ & 0.990 \\
\hline Diastolic Blood Pressure, $\mathrm{mm} \mathrm{Hg}$ & $81 \pm 13$ & $83 \pm 13$ & 0.396 & $81 \pm 13$ & $78 \pm 9$ & 0.140 \\
\hline Active smoking & $19(39)$ & $27(29)$ & 0.256 & $19(39)$ & $38(39)$ & $>0.999$ \\
\hline Diabetes mellitus II & $3(6)$ & $4(4)$ & 0.693 & $3(6)$ & $5(5)$ & $>0.999$ \\
\hline Family history of CAD & $16(33)$ & $43(46)$ & 0.152 & $16(33)$ & $27(28)$ & 0.566 \\
\hline PROCAM risk score & $9(4-21)^{\mathrm{a}}$ & $7(2-16)^{b}$ & 0.131 & $9(4-21)^{\mathrm{a}}$ & $8(3-16)$ & 0.280 \\
\hline
\end{tabular}

Continous variables are described as mean $( \pm \mathrm{SD})$ or as median (interquartile range); categorical variables as number $(\%)$

$B M I$ body mass index

${ }^{\mathrm{a}} n=19$ lost to PROCAM risk score; ${ }^{\mathrm{b}} n=34$ lost to PROCAM risk score

nosis is crucial, whereby the risk of death, recurrence, as well as life-threatening events should be considered [2, 4].

Within the current European Society of Cardiology Guidelines for the diagnosis and management of syncope, only limited guidance is offered for diagnostic strategies to detect CAD [2]. Ischaemia evaluation is recommended within the current guidelines of the American College of Cardiology/American Heart Association (ACC/AHH) for syncope patients with known, or who are at risk for, coronary artery disease (CAD) [6]. However, there is no guidance regarding anatomical imaging techniques to detect $\mathrm{CAD}$ in syncope patients despite the evidence that arrhythmic causes for syncope, such as atrial fibrillation or ventricular tachycardia, have a higher risk of major adverse cardiovascular and cerebral events in the presence of CAD [7, 8]. Additionally, all patients with syncope and ischaemic heart disease have an increased risk of death $[1$, 3,9].

Currently, coronary computed tomography (CT) angiography (CCTA) is a widely implemented non-invasive imaging modality to diagnose CAD [10-12]. Conventional CCTA reading includes assessment of the coronary calcium score (CCS), luminal stenosis severity and extent of CAD with high sensitivity and specificity $[10,12]$. It may be considered in stable chest pain patients with an intermediate pre-test probability of ischaemic heart disease [13]. Despite the wide use of CCTA in patients with stable chest pain, there are no recommendations for CAD detection with CCTA in patients presenting with syncope. Nevertheless, diagnosing $\mathrm{CAD}$ within syncope patients in an early stage could have important prognostic and therapeutic clinical implications. Therefore, the aim of the present study was to investigate the presence and extent of CAD, as defined by
CCTA, in patients presenting with syncope at the outpatient cardiology clinic.

\section{Methods}

\section{Study population}

This was an observational single-centre study analysing 142 retrospectively collected consecutive patients presenting with syncope at the outpatient cardiology department between May 2007 and April 2015 who were referred for CCTA within their diagnostic workup. Patients were selected if they met the definition of syncope, which was defined as transient loss of consciousness due to global cerebral hypoperfusion with rapid onset, short duration and spontaneous complete recovery [2].

General exclusion criteria for CCTA examination were: haemodynamic instability, pregnancy, renal insufficiency (defined as glomerular filtration rate $<45 \mathrm{ml} / \mathrm{min} / 1.73 \mathrm{~m}$ ) and known severe allergic reactions regarding iodine.

This study was approved by the Institutional Review Board (METC 15-4-091) and complies to the ethical guidelines of the 1975 Declaration of Helsinki. Written informed consent was waived, because the data were anonymously recorded and analysed in accordance with guidelines of our Review Board.

\section{Data collection and definitions}

Data regarding syncope type, age, gender, cardiovascular risk factors, medication use, additional diagnostic testing and CCTA results were collected from the patients' charts and electronic medical records. 
Table 2 Distribution of conventional CT parameters across cardiac syncope patients versus non-cardiac syncope patients and matched chest pain control group

\begin{tabular}{|c|c|c|c|c|c|c|}
\hline \multirow[b]{2}{*}{ CT parameters } & \multicolumn{3}{|c|}{$\begin{array}{l}\text { Cardiac syncope vs non-cardiac syncope } \\
n=142\end{array}$} & \multicolumn{3}{|c|}{$\begin{array}{l}\text { Cardiac syncope vs chest pain control group } \\
n=147\end{array}$} \\
\hline & $\begin{array}{l}\text { Cardiac } \\
\text { syncope } \\
n=49\end{array}$ & $\begin{array}{l}\text { Non-cardiac } \\
\text { syncope } \\
n=93\end{array}$ & $P$-value & $\begin{array}{l}\text { Cardiac } \\
\text { syncope } \\
n=49\end{array}$ & $\begin{array}{l}\text { Chest pain } \\
\text { control } \\
\text { group } \\
n=98\end{array}$ & $P$-value \\
\hline Presence and extent of CAD & - & - & 0.001 & - & - & 0.133 \\
\hline - no CAD & $14(28)$ & $49(53)$ & - & $14(28)$ & $36(37)$ & - \\
\hline - mild CAD, $<50 \%$ luminal stenosis & $15(31)$ & $24(26)$ & - & $15(31)$ & $25(26)$ & - \\
\hline - moderate CAD, 50-70\% luminal stenosis & $4(8)$ & $13(14)$ & - & $4(8)$ & $18(18)$ & - \\
\hline - severe $\mathrm{CAD},>70 \%$ luminal stenosis & $16(33)$ & $7(7)$ & - & $16(33)$ & $19(19)$ & - \\
\hline Coronary calcium score, $A U$ & $80(0-387)$ & $0(0-79)$ & 0.002 & $80(0-387)$ & $36(0-205)$ & 0.272 \\
\hline Segment involvement score, $0-16$ & $2(0-5)$ & $0(0-2)$ & 0.004 & $2(0-5)$ & $2(0-4)$ & 0.364 \\
\hline Segment stenosis score, $0-48$ & $3(0-7)$ & $0(0-3)$ & 0.003 & $3(0-7)$ & $2(0-7)$ & 0.477 \\
\hline
\end{tabular}

$C T$ computed tomography, $C A D$ coronary artery disease, $A U$ Agatston Unit

The type of syncope was ascertained by chart review by two reviewers (N.M., T.D.), blinded for CCTA results. The definitive syncope type was ascertained by consensus between the two reviewers. The following pathophysiological classification and sub-classification was used to adequately define the syncope types in each individual patient [2]:

1. Cardiac syncope (cardiovascular): arrhythmia or structural cardiopulmonary disease as primary cause,

2. Reflex (neurally-mediated) syncope: vasovagal, situational or carotid sinus syncope,

3. Syncope due to orthostatic hypotension: primary or secondary autonomic failure, drug-induced orthostatic hypotension or volume depletion,

4. Syncope of unknown cause: defined as an unknown cause despite additional diagnostic testing.

Subsequently, patients with reflex syncope, orthostatic syncope and syncope of unknown cause were classified as having 'non-cardiac syncope', which led to two main syncope categories: cardiac and non-cardiac syncope.

Diabetes mellitus was defined as fasting glucose levels of $\geq 7 \mathrm{mmol} / \mathrm{l}$ or treatment with either diet intervention, oral glucose lowering agent or insulin [14]; smoking was defined as current smoking. A positive family history was defined as having a first-degree relative with a history of myocardial infarction or sudden cardiac death before the age of 60 .

The PROCAM risk score was determined according to the following parameters: age, LDL cholesterol, smoking, HDL cholesterol, systolic blood pressure, family history of premature myocardial infarction, diabetes mellitus, and triglycerides [15]. This risk score predicts the absolute 10year risk for the occurrence of an acute coronary event (fatal or non-fatal myocardial infarction or acute coronary death). A score $<10 \%$ is estimated as low risk, $10-20 \%$ as intermediate risk, and $>20 \%$ as high risk.

\section{Control population}

To further study the impact of cardiac syncope symptoms on detection of CAD, we compared the cardiac syncope patients with stable chest pain patients. The cardiac syncope patients were compared (1:2 ratio) with stable chest pain patients, referred for CCTA from the outpatient cardiology clinic to compare the prevalence and extent of CAD in cardiac syncope patients with stable chest pain patients. Matching was based upon age, gender, smoking status, diabetes mellitus type 2 and systolic blood pressure $( \pm 10 \mathrm{~mm} \mathrm{Hg})$.

\section{CCTA acquisition}

Scans were performed using a 64-slice multidetector computed tomography scanner (Brilliance 64; Philips Healthcare, Best, The Netherlands) or a $2^{\text {nd }}$ generation dual-source CT scanner (Somatom Definition Flash, Siemens Healthcare, Forchheim, Germany). Data acquisition parameters for the Brilliance 64 were a $64 \times 0.625 \mathrm{~mm}$ slice collimation, a gantry rotation time of $420 \mathrm{~ms}$ and a tube voltage of 80 or $120 \mathrm{kV}$ depending on patients' height and weight. Data acquisition parameters for the Somatom Definition Flash were a $2 \times 128 \times 0.600 \mathrm{~mm}$ slice collimation, a gantry rotation time of $280 \mathrm{~ms}$ and a tube voltage of 100 or $120 \mathrm{kV}$ depending on patients' height and weight. A noncontrast enhanced scan was performed using $120 \mathrm{kV}$ and $3 \mathrm{~mm}$ slice thickness to determine the CCS [16]. CCTA was performed using 75-120 ml of contrast agent (Xenetix 350; Guerbet, France or Ultravist 300; Bayer Healthcare, Berlin, Germany) injected in the antecubital vein at a rate of $5.2-7.4 \mathrm{ml} / \mathrm{s}$ followed by $40 \mathrm{ml}$ intravenous saline at the same flow rates.

Scan protocols differed between both CT scanners. For the 64-slice scanner, a prospectively gated 'Step and shoot' 


\section{Advertisement placed here.}

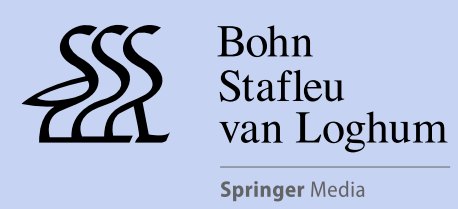

Houten 2017 


\section{Advertisement placed here.}

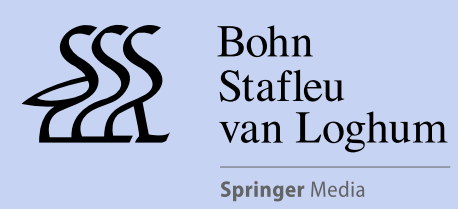

Houten 2017 


\section{Advertisement placed here.}

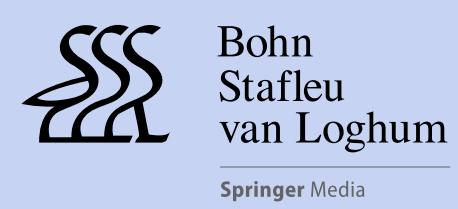

Houten 2017 


\section{Advertisement placed here.}

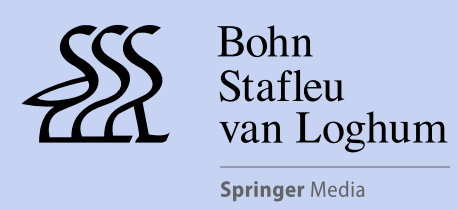

Houten 2017 
Fig. 1 CAD presence and extent (percentages) within cardiac syncope patients versus non-cardiac syncope patients $p$-value $=0.001$

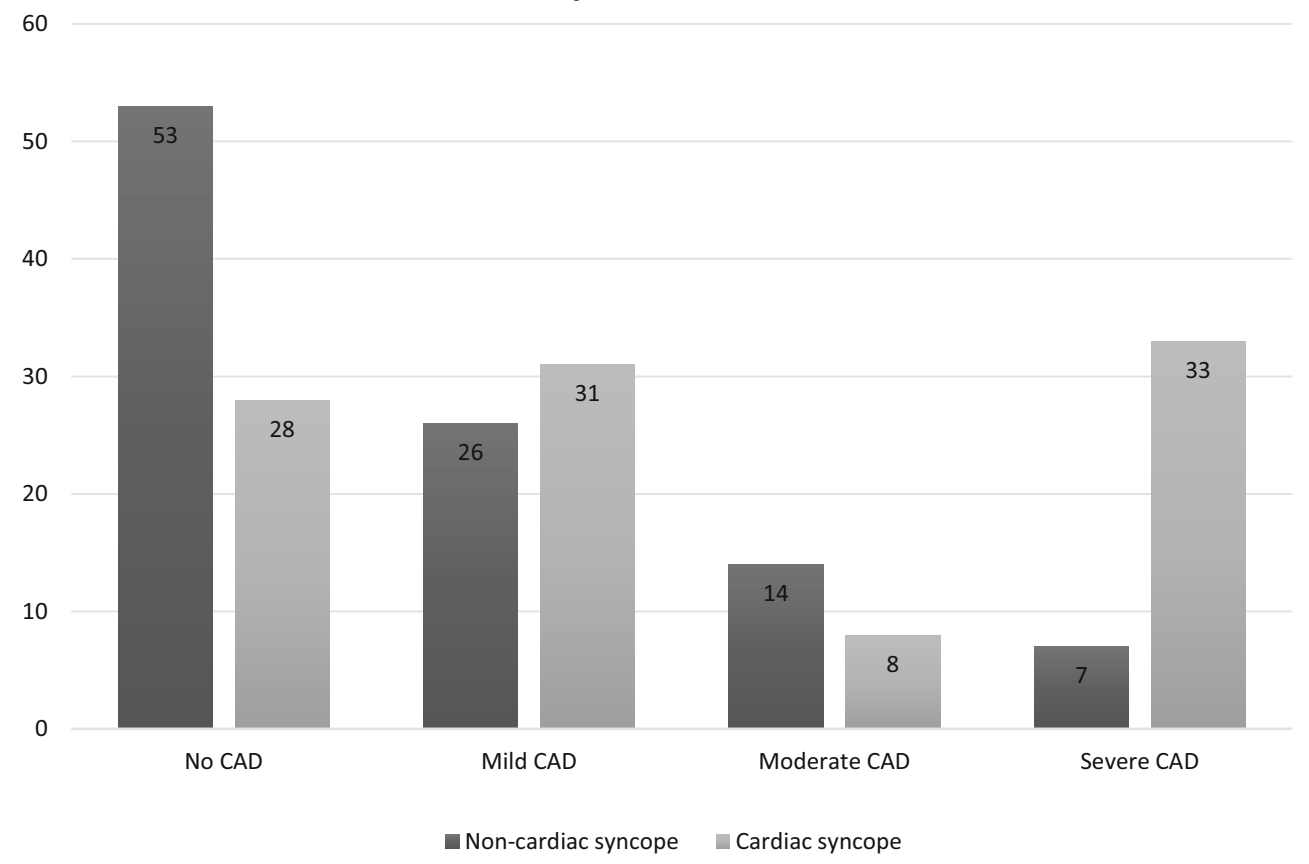

protocol was used in patients with stable heart rate $<65 \mathrm{bpm}$. In patients with a heart rate $>65 \mathrm{bpm}$, a retrospectively gated 'Helical' protocol was used with dose modulation. For the $2^{\text {nd }}$ generation dual-source CT scanner, a prospectively gated high-pitch spiral 'Flash' protocol was used in patients with a stable heart rate $<60 \mathrm{bpm}$. In patients with a stable heart rate between $60-90 \mathrm{bpm}$, a prospectively gated axial 'Adaptive sequence' protocol was used. In patients with a heart rate $>90 \mathrm{bpm}$ or with an irregular heart rhythm, a retrospectively gated 'Helical' protocol was used. Dose modulation was switched on in all three protocols using tube current modulation (CARE Dose4D, Siemens Healthcare, Forchheim, Germany).

Patients received $50 \mathrm{mg}$ Metoprolol tartrate orally (AstraZeneca, Zoetermeer, the Netherlands), two hours before CCTA, unless contra-indicated. If indicated, an additional dose of 5-20 mg Metoprolol was administered intravenously to lower the heart rate. All patients received nitroglycerine (Pohl-Boskamp, Hohenlockstedt, Germany) sublingually in a dose of $0.8 \mathrm{mg}$ just prior to CCTA.

\section{CCTA assessment}

The Agatston method was used to define the CCS [16]. CCTA's were independently analysed by a cardiologist and a radiologist, both experienced in the assessment of CCTA. In case of disagreement, consensus was reached by discussion. The assessment was performed using the source images on the provided software (Cardiac Comprehensive Analysis, Philips Healthcare or Syngo CT 2010A,
Siemens Healthcare). The coronary artery tree was analysed for the presence and extent of CAD, according to the 16-segment classification of the American Heart Association [17]. Plaques were defined as visible structures within or adjacent to the coronary artery lumen, which could be clearly distinguished from the vessel lumen and the surrounding pericardial tissue. The degree of stenosis was visually defined and classified as no CAD (no luminal stenosis), mild ( $<50 \%$ luminal stenosis), moderate (50-70\% luminal stenosis), severe ( $>70 \%$ luminal stenosis). A segment involvement score was defined by counting all coronary segments with plaques (irrespective of degree of stenosis), which resulted in a score ranging from 0-16 [18]. A segment stenosis score was the sum of the lesion severity in all 16 coronary segments, resulting in a score ranging from $0-48$ [18].

\section{Statistical analysis}

Data were analysed using SPSS version 23.0 (SPSS Inc., Chicago, IL, USA). Continuous variables were checked whether they were normally distributed using box plots, histograms or by computing skewness and kurtosis. Continuous data were reported as means and standard deviations (SDs) if normally distributed. If data were not normally distributed, continuous data were reported as medians and boundaries of interquartile ranges (IQR). Proportions (\%) were used for categorical values.

Differences across groups were assessed using the independent $t$-test for normally distributed data after performing 
Fig. 2 CAD presence and extent (percentages) within cardiac syncope versus matched chest pain control group $p$-value $=0.133$

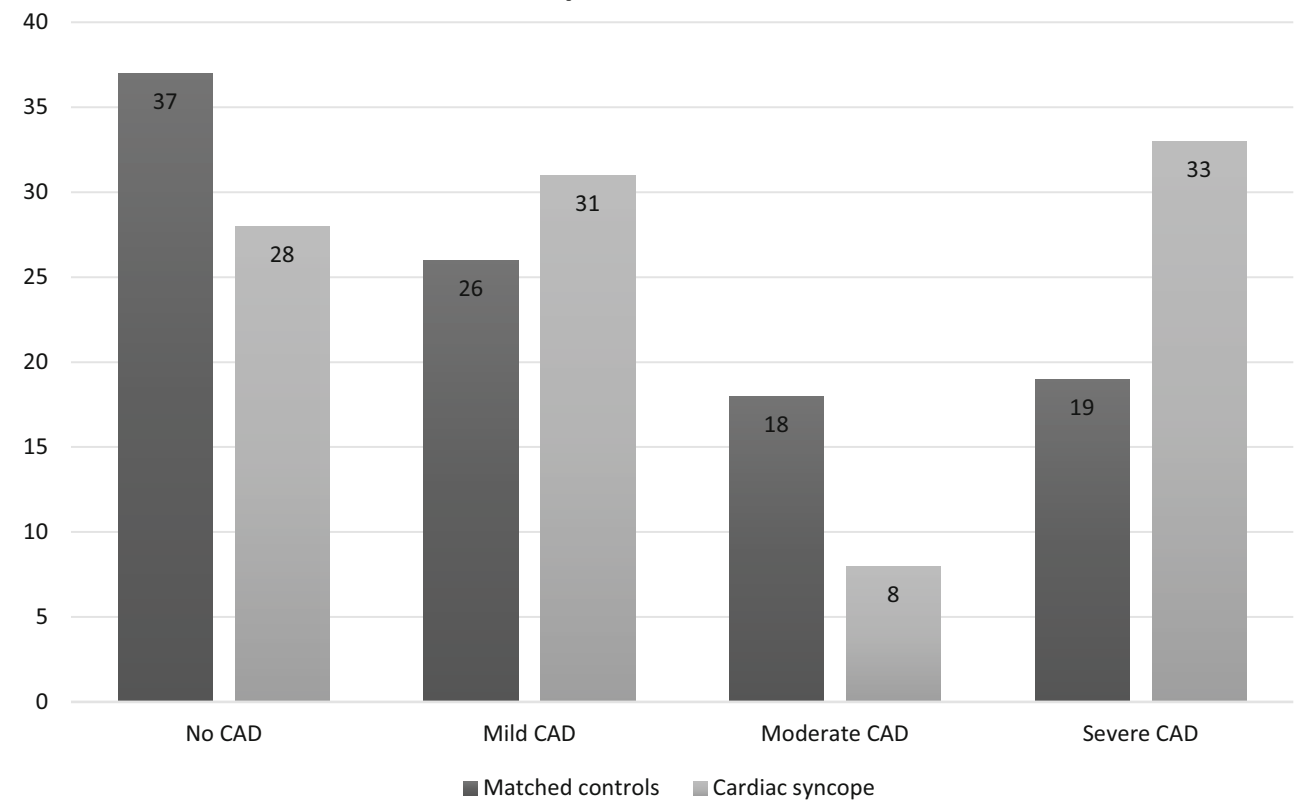

Levene's test for equality of variance. The Mann-Whitney test was used for data that were not normally distributed. Categorical variables were tested with Fisher's exact test.

All $p$-values were 2 -sided, and a $p$-value below 0.05 was considered statistically significant.

\section{Results}

\section{Study population}

Between May 2007 and April 2015, 142 consecutive patients presented with syncope at the outpatient cardiology clinic who were referred for CCTA. The distribution of syncope classifications was as follows: $49(35 \%)$ cardiac syncope, 63 (44\%) reflex (neurally-mediated) syncope, 10 (7\%) orthostatic syncope and $20(14 \%)$ syncope of unknown cause. Subsequently, patients with reflex syncope, orthostatic syncope and syncope of unknown cause were classified as having 'non-cardiac syncope' leading to two main categories, with $49(35 \%)$ cardiac syncope patients and $93(65 \%)$ non-cardiac syncope patients.

The baseline characteristics of the study population are described in Table 1. When compared to non-cardiac syncope patients, only age showed a statistically significant difference between the cardiac and non-cardiac syncope groups (mean (SD): 60 (13) versus $54(12) ; p=0.002$ ). The Online Supplemental Material provides further detailed clinical information regarding the syncope patients.

\section{Presence and extent of CAD in syncope}

The distribution of CAD presence and extent in cardiac and non-cardiac syncope patients was as follows: $72 \%$ versus $48 \%$ any $\mathrm{CAD} ; 31 \%$ versus $26 \%$ mild CAD; $8 \%$ versus $14 \%$ moderate $\mathrm{CAD}$ and $33 \%$ versus $7 \%$ severe CAD (Table 2 ).

Fig. 1 visualises $\mathrm{CAD}$ prevalence and distribution of CAD in patients with cardiac and non-cardiac syncope. CAD presence and extent was significantly higher in patients with cardiac syncope in comparison with non-cardiac syncope patients (Table $1 ; p=0.001$ ). Additionally, all conventional CT parameters including CCS, segment involvement and segment stenosis score were significantly higher in patients with cardiac syncope compared to noncardiac syncope (80 [0-387] versus 0 [0-79]; 2 [0-5] versus 0 [0-2]; 3 [0-7] versus 0 [0-3]) (all $p$-values $\leq 0.004$; Table 2).

\section{Presence and extent of CAD in patients with cardiac syncope versus matched chest pain controls}

Fig. 2 displays the presence and extent of CAD in both cardiac syncope patients and matched chest pain controls. Patients with cardiac syncope showed a higher prevalence of any CAD (72\% versus $63 \%$, respectively) and percentage of severe luminal stenosis (33\% versus 19\%). Interestingly, no statistically significant difference was observed between cardiac syncope patients and chest pain controls regarding overall prevalence of CAD presence and its extent ( $p=$ $0.133)$. 
Table 2 shows that all other conventional CT parameters including CCS, segment involvement score and segment stenosis score were comparable between cardiac syncope patients and chest pain controls (all $p$-values $\geq 0.272$ ).

\section{Discussion}

To our best knowledge, this is the first study dedicated to investigating the prevalence and extent of CAD in patients presenting with syncope. The main finding of this study was that cardiac syncope patients showed a high presence and extent of CAD in comparison with non-cardiac syncope patients. In addition, all other coronary CT parameters like CCS, segment involvement score and stenosis score were also significantly higher in cardiac syncope patients compared to non-cardiac syncope patients. When compared to stable chest pain controls, patients with cardiac syncope showed a higher, however, non-significant, prevalence of any CAD (72\% versus $63 \%$, respectively) and percentage of severe luminal stenosis (33\% versus 19\%). Taken together, these results suggest that the non-invasive evaluation of CAD, using CCTA, could be considered within the diagnostic workup of patients presenting with cardiac syncope at the outpatient cardiology clinic. Additionally, CCTA may show alternative causes for cardiovascular syncope such as congenital anomalies of coronary arteries, hypertrophic cardiomyopathy, pulmonary embolism, obstructive valvular heart disease, intracardiac masses and pericardial diseases.

\section{Association and relevance of CAD in cardiac syncope}

Meticulous history taking is essential in arriving at a diagnosis in patients with syncope. Once cardiac syncope is suspected, a wide range of different diagnostic tests can be considered in patients presenting with syncope [2, 6]. However, there is no guidance regarding anatomical imaging techniques to detect $\mathrm{CAD}$ due to lacking evidence describing the extent and nature of CAD in patients with cardiac syncope. The relationship between cardiac ischaemia and syncope is multiple, including induction of non-sustained ventricular arrhythmias and sinoatrial or atrioventricular block, or by triggering e.g. the Bezold-Jarisch-reflex causing severe bradycardia and hypotension. Apart from a direct relationship, indirect mechanisms may be important, including old myocardial infarction with ventricular remodelling as a basis for re-entrant or adrenergic ventricular tachycardia; likewise, atrial remodelling leading to late onset atrioventricular nodal tachycardia or atrial tachycardias may at times occur with well-known haemodynamic compromise eliciting syncope at the beginning of the attack [19]. In all of the above-mentioned aetiologies, CAD may be causal or contributory for syncope whereby one could conjecture that interventional and vascular prophylactic management may help to reduce further syncope in these patients. In all other cases, CAD presence should be considered as coincidental wherefore vascular prophylactic vascular management may not be indicated in the management of syncope.

\section{Current guidance for the detection of CAD in cardiac syncope}

Within the European Society of Cardiology guidelines for the diagnosis and management of syncope, exercise stress testing is only recommended in patients with suspected exercise-induced syncope, which concerns a rare condition [2]. Concurrently, ischaemia evaluation is recommended by the American College of Cardiology/American Heart Association (ACC/AHA) for patients with syncope and an intermediate-to-high risk for coronary heart disease or known CAD, but such strategy may underdiagnose the presence of non-obstructive CAD, which still is associated with high major adverse cardiac event rates [6, 20, 21]. On the other hand, a previous report revealed a low-diagnostic yield for stress myocardial perfusion imaging across all risk categories in syncope patients without known CAD [22].

\section{Prior studies}

Previously, Soteriades et al. investigated the incidence and prognosis of syncope among participants of the Framingham Heart Study and found that patients with a cardiac syncope were more likely to have a history of CAD and were at an increased risk for death from any cause and cardiovascular events [3]. A more recent study of patients presenting with syncope at the emergency department with trauma, has shown that patients with a history of CAD are four times more likely to have cardiac syncope in contrast to non-cardiac syncope [23].

These previous reports support the high prevalence and extent of CAD in patients presenting with cardiac syncope compared to patients with non-cardiac syncope within the present study. Furthermore, in the presence of obstructive CAD in patients with syncope, treatment by either percutaneous coronary intervention (PCI) or medical management did not improve readmission rates due to syncope. However, PCI did improve long-term mortality in patients with syncope, suggesting the need for imaging of the coronary arteries [24].

Moreover, in syncope patients with left ventricular dysfunction, inducible ventricular tachycardia was frequent in the presence of CAD and associated with a bad prognosis [7]. Therefore, by diagnosing stable CAD and providing additional treatment with vascular protective medication, 
the prognosis of cardiac syncope patients could be positively influenced. HMG-CoA reductase inhibitors (statins) have become a cornerstone in the treatment of patients with stable CAD due to their lipid-lowering characteristics and additional atherosclerotic plaque stabilization, systemic inflammation and thrombogenicity reducing effects [25]. In line with these findings, recent review articles summarise that statins even reduce the incidence of ventricular tachycardia/fibrillation and sudden cardiac death in patients with CAD due to their anti-ischaemic, and possibly also by their antiarrhythmic or anti-inflammatory, effects [26, 27].

\section{Study limitations}

This study has several limitations that should be mentioned. Firstly, it concerns a study with a relatively small sample size. Secondly, there was some degree of referral bias considering that our institution is a tertiary centre for patients with syncope. This is confirmed by the fact that within our syncope study population, a higher relative prevalence of cardiac syncope was observed in comparison to previous reports $[2,3]$. Thirdly, the syncope patients were included if they were referred for CCTA, inducing some degree of selection bias. The combination of referral as well as selection bias could have contributed to the high prevalence and extent of CAD within the cardiac syncope patients. Also important is the fact that no direct causal relationships could be identified regarding the presence and extent of CAD and syncope due to the present study design, as this would require a prospective interventional study.

\section{Conclusions}

Patients with cardiac syncope show a high presence and extent of CCTA defined CAD in contrast to patients with non-cardiac syncope. These results suggest that CAD may play an important role in the occurrence of cardiac syncope and should be considered in the diagnostic workup and treatment of syncope patients.

Conflict of interest S. Altintas, T. Dinh, N.G.H.M. Marcks, M. Kok, A.J.J. Aerts, B. Weijs, Y. Blaauw, J.E. Wildberger, M. Das, B.L.J.H. Kietselaer and H.J.G.M. Crijns declare that they have no competing interests.

Open Access This article is distributed under the terms of the Creative Commons Attribution 4.0 International License (http:// creativecommons.org/licenses/by/4.0/), which permits unrestricted use, distribution, and reproduction in any medium, provided you give appropriate credit to the original author(s) and the source, provide a link to the Creative Commons license, and indicate if changes were made.

\section{References}

1. Kapoor WN. Syncope. N Engl J Med. 2000;343:1856-62.

2. Moya A, Sutton R, Ammirati F, et al. Guidelines for the diagnosis and management of syncope (version 2009). Eur Heart J. 2009;30:2631-71.

3. Soteriades ES, Evans JC, Larson MG, et al. Incidence and prognosis of syncope. N Engl J Med. 2002;347:878-85.

4. Kapoor WN. Current evaluation and management of syncope. Circulation. 2002;106:1606-9.

5. Kessler C, Tristano JM, De Lorenzo R. The emergency department approach to syncope: evidence-based guidelines and prediction rules. Emerg Med Clin North Am. 2010;28:487-500.

6. Strickberger SA, Benson DW, Biaggioni I, et al. AHA/ACCF scientific statement on the evaluation of syncope: from the American Heart Association Councils on Clinical Cardiology, Cardiovascular Nursing, Cardiovascular Disease in the Young, and Stroke, and the Quality of Care and Outcomes Research Interdisciplinary Working Group; and the American College of Cardiology Foundation In Collaboration With the Heart Rhythm Society. J Am Coll Cardiol. 2006;47:473-84.

7. Brembilla-Perrot B, Suty-Selton C, Beurrier D, et al. Differences in mechanisms and outcomes of syncope in patients with coronary disease or idiopathic left ventricular dysfunction as assessed by electrophysiologic testing. J Am Coll Cardiol. 2004;44:594-601.

8. Lip GY, Nieuwlaat R, Pisters R, Lane DA, Crijns HJ. Refining clinical risk stratification for predicting stroke and thromboembolism in atrial fibrillation using a novel risk factor-based approach: the euro heart survey on atrial fibrillation. Chest. 2010;137:263-72.

9. Racco F, Sconocchini C, Alesi C, Zappelli L, Pratillo G. Long-term follow-up after syncope. A group of 183 patients observed for 5 years. Minerva Cardioangiol. 2000;48:69-78.

10. Budoff MJ, Dowe D, Jollis JG, et al. Diagnostic performance of 64-multidetector row coronary computed tomographic angiography for evaluation of coronary artery stenosis in individuals without known coronary artery disease: results from the prospective multicenter ACCURACY (Assessment by Coronary Computed Tomographic Angiography of Individuals Undergoing Invasive Coronary Angiography) trial. J Am Coll Cardiol. 2008;52:1724-32.

11. Meijboom WB, Meijs MF, Schuijf JD, et al. Diagnostic accuracy of 64-slice computed tomography coronary angiography: a prospective, multicenter, multivendor study. J Am Coll Cardiol. 2008;52:2135-44.

12. Miller JM, Rochitte CE, Dewey M, et al. Diagnostic performance of coronary angiography by 64-row CT. N Engl J Med. 2008;359:2324-36.

13. Fihn SD, Gardin JM, Abrams J, et al. ACCF/AHA/ACP/AATS/ PCNA/SCAI/STS Guideline for the diagnosis and management of patients with stable ischemic heart disease: a report of the American College of Cardiology Foundation/American Heart Association Task Force on Practice Guidelines, and the American College of Physicians, American Association for Thoracic Surgery, Preventive Cardiovascular Nurses Association, Society for Cardiovascular Angiography and Interventions, and Society of Thoracic Surgeons. J Am Coll Cardiol. 2012;2012(60):e44-e164.

14. Expert Committee on the Diagnosis and Classification of Diabetes Mellitus. Report of the expert committee on the diagnosis and classification of diabetes mellitus. Diabetes Care. 2003;26(Suppl 1):S5-20.

15. Assmann G, Cullen P, Schulte H. Simple scoring scheme for calculating the risk of acute coronary events based on the 10-year followup of the prospective cardiovascular Munster (PROCAM) study. Circulation. 2002;105:310-5.

16. Agatston AS, Janowitz WR, Hildner FJ, Zusmer NR, Viamonte M Jr., Detrano R. Quantification of coronary artery calcium using ul- 
trafast computed tomography. J Am Coll Cardiol. 1990;15(4):82732.

17. Austen WG, Edwards JE, Frye RL, et al. A reporting system on patients evaluated for coronary artery disease. Report of the Ad Hoc Committee for Grading of Coronary Artery Disease, Council on Cardiovascular Surgery, American Heart Association. Circulation. 1975;5:5-40.

18. Min JK, Shaw LJ, Devereux RB, et al. Prognostic value of multidetector coronary computed tomographic angiography for prediction of all-cause mortality. J Am Coll Cardiol. 2007;50:1161-70.

19. Pentinga ML, Meeder JG, Crijns HJ, de Muinck ED, Wiesfeld AC, Lie KI. Late onset atrioventricular nodal tachycardia. Int J Cardiol. 1993;38:293-8.

20. Hendel RC, Berman DS, Di Carli MF, et al. ACCF/ASNC/ACR/ AHA/ASE/SCCT/SCMR/SNM 2009 Appropriate Use Criteria for Cardiac Radionuclide Imaging: A Report of the American College of Cardiology Foundation Appropriate Use Criteria Task Force, the American Society of Nuclear Cardiology, the American College of Radiology, the American Heart Association, the American Society of Echocardiography, the Society of Cardiovascular Computed Tomography, the Society for Cardiovascular Magnetic Resonance, and the Society of Nuclear Medicine. J Am Coll Cardiol. 2009;53:2201-29.

21. Nakazato R, Arsanjani R, Achenbach S, et al. Age-related risk of major adverse cardiac event risk and coronary artery disease extent and severity by coronary CT angiography: results from 15187 patients from the International Multisite CONFIRM Study. Eur Heart J Cardiovasc Imaging. 2014;15:586-94.

22. AlJaroudi WA, Alraies MC, Wazni O, Cerqueira MD, Jaber WA. Yield and diagnostic value of stress myocardial perfusion imaging in patients without known coronary artery disease presenting with syncope. Circ Cardiovasc Imaging. 2013;6:384-91.

23. Bhat PK, Pantham G, Laskey S, Como JJ, Rosenbaum DS. Recognizing cardiac syncope in patients presenting to the emergency department with trauma. J Emerg Med. 2014;46:1-8.

24. Anderson LL, Dai D, Miller AL, Roe MT, Messenger JC, Wang TY. Percutaneous coronary intervention for older adults who present with syncope and coronary artery disease? Insights from the National Cardiovascular Data Registry. Am Heart J. 2016;176:1-9.

25. Task Force M, Montalescot G, Sechtem U, et al. ESC guidelines on the management of stable coronary artery disease: the Task Force on the management of stable coronary artery disease of the European Society of Cardiology. Eur Heart J. 2013;2013(34):2949-3003.

26. Beri A, Contractor T, Khasnis A, Thakur R. Statins and the reduction of sudden cardiac death: antiarrhythmic or anti-ischemic effect? Am J Cardiovasc Drugs. 2010;10:155-64.

27. Liao JK, Laufs U. Pleiotropic effects of statins. Annu Rev Pharmacol Toxicol. 2005;45:89-118. 